Diasolid S, 80-100 mesh, 10\% Polydiethylenglycolsuccinate.

2) Carrier gas: Herium $1.7 \mathrm{Kg} / \mathrm{cm}^{2}$.

3) Columntemperature : $200^{\circ} \mathrm{C}$

4) Sample temperature: $270-300^{\circ} \mathrm{C}$

$50 \mathrm{mg} / \mathrm{dl}$ of Arachidic acid to be soluble in Methanol as standard, was added to the sample ( $4 \mathrm{ml}$ of CSF), and after saponifing, the sample was analyzed. The quantitative error of the recovery test for Arachidic acid was lesser than $\pm 5 \%$.

\title{
Result
}

1) normal group

The constitutions of fatty acids in CSF were remarkably different from that in serum. The fatty acids, has lesser carbon number than Palmitic were few in serum, and much in CSF.

On the contrary, the fatty acids, range over C16, were of large quantity in serum, and few in CSF.

It was found that an unkown fatty acid existed between Palmitic acid and Myristic acid in CSF.

2) brain tumor group.

Qualitatively, the constitutions of CSF-fatty acids were the same as that in normal CSF. However the quantity of CSF-fatty acids was diminished.

\section{S-A-5. Lipid and it's Fatty Acid Composition of Brain Tumors}

\author{
Yasushi Tatsuzawa, Masahiro Kato, Tsuneo Hasegawa \\ and Toyozo Aizawa \\ Department of Internal Medicine, Keio University \\ Shoji IsHIMORI \\ Department of Surgery, Keio University
}

By the methods of thin-layer chromatography, gas-liquid chromatography and biochemical assay, the lipid and it's fatty acid composition were analyzed in the following materials; glioblastoma multiforme (solid and soft area), astrocytoma, oligodendroglioma, two cases of meningioma, neurofibroma, craniopharyngioma and also grey and white matter as the controls.

Phospholipid content of tumors showed lower level than that of normal grey matter, but no significant differences were found among tumors. 
Total cholesterol was slightly decreased in glioblastoma and astrocytoma and markedly decreased in other tumors.

Among the phospholipid fractions, phosphatidyl ethanolamine was decreased much more than the remainder of phospholipids, namely phosphatidyl choline, sphingomyelin and phosphatidyl serine. Lysolecithin was present in a small content in meningioma, neurofibroma and craniopharyngioma.

Cerebroside and sulfatide were extremely diminished or absent in tumors of the central nervous system.

Ratio of free cholesterol to esterified one was inconsistent among the tumors, and even in the same tumor the ratio was reversed between it's solid and soft areas.

In fatty acid composition, odd-numbered fatty acids which is very small in amount in the normal grey and white matter, were present in sphingomyelin, cholesterol ester triglyceride and non-esterified fatty acids. Linoleic acid, which is also present in a small amount in the normal brain, was found in the fraction of phosphatidyl choline, cholesterol ester and triglyceride. Polyunsaturated long-chain fatty acids were dimished in cerebroside of tumors.

There was a considerably large amount of unidentified fatty acid (retention time approx. $52 \mathrm{~min}$ ) was contained in cholesterol ester.

Fatty acid composition of solid area in the glioblastoma multiforme was closely similar to that of soft area, however lipid composition and the ratio of cholesterol ester to free one was different between them.

There are some differences between glioma and craniopharyngioma, neurofibroma, in the fatty acid composition, but a case of meningioma revealed the similar fatty acid composition with glioma.

\title{
S-A-6. The Relationship between the Enzymatic Activities of Human Brain Tumors and Clinical Features
}

\author{
Takao Kawashima, Kisaburo Takeuchi, Masato Nakamura, \\ Takuro Ogata and Akira Nishimoto \\ Department of Neurological Surgery, School of Medicine, Okayama University
}

A histochemical observation on human brain tumors of 126 cases was made on hydrolytic enzymes such as Alkaline phosphatase (ALP), Acid phosphatase (ACP), $\beta$-Esterase ( $\beta$-EST), Leucine aminopeptidase (LAP) and $\beta$-Glucuronidase $(\beta-G L)$, and on dehydrogenases, Succinic dehydrogenase 